\title{
Model as You Do: Engaging an S-BPM Vendor on Process Modelling in 3D Virtual Worlds
}

\author{
Joel Harman, Ross Brown, Udo Kannengiesser, \\ Nils Meyer and Thomas Rothschädl
}

\begin{abstract}
Accurate process model elicitation continues to be a time-consuming task, requiring skill on the part of the interviewer to extract explicit and tacit process information from the interviewee. Many errors occur in this elicitation stage that would be avoided by better activity recall, more consistent specification methods and greater engagement in the elicitation process by interviewees. Metasonic $\mathrm{GmbH}$ has developed a process elicitation tool for their process suite. As part of a research engagement with Metasonic, staff from QUT, Australia have developed a $3 \mathrm{D}$ virtual world approach to the same problem, viz. eliciting
\end{abstract}

\footnotetext{
J. Harman ( $\square)$

Science and Engineering Faculty, Queensland University of Technology,

2 George St., Brisbane, QLD 4000, Australia

e-mail: joel.harman@connect.qut.edu.au

R. Brown

Information Systems School, Science and Engineering Faculty, Queensland University

of Technology, 2 George St., Brisbane, QLD 4000, Australia

e-mail: r.brown@qut.edu.au

U. Kannengiesser

Metasonic GmbH, Münchner Straße 29, 85276 Pfaffenhofen, Germany

e-mail: udo.kannengiesser@metasonic.de

N. Meyer

Research Development, Metasonic GmbH, Münchner Straße 29,

85276 Pfaffenhofen, Germany

e-mail: nils.meyer@metasonic.de

T. Rothschädl

Ruxit, Blütenstraße 14, 4040 Linz, Austria

e-mail: Thomas.rothschaedl@ ruxit.com
} 
process models from stakeholders in an intuitive manner. This book chapter tells the story of how QUT staff developed a 3D Virtual World tool for process elicitation and took the outcomes of their research project to Metasonic for evaluation, and of Metasonic's response to the initial proof of concept.

\subsection{Metasonic Engages QUT June 2013}

Metasonic $\mathrm{GmbH}$, a German business process management vendor, has implemented a complete process management suite called Metasonic Suite and a process elicitation tool called Metasonic Touch, developed from a previous university research project (Oppl and Stary 2011). Metasonic sought to engage with researchers at QUT to explore new areas of innovation around the effectiveness of their software tools. After a brief phone call with Dr. Albert Fleischmann, director and co-founder of Metasonic, Dr. Ross Brown pitched the idea of using virtual worlds in the task of process elicitation. Metasonic accepted this proposal, a scholarship was offered to QUT by the company, and an IT Honours student, Joel Harman, was taken on in December 2013 to begin the twelve-month-long research project.

Process model elicitation still poses a huge challenge with respect to the quality of the resulting process models, independently of whether the information was gathered from interviews (Kabicher and Rinderle-Ma 2011), by exploiting existing data sources (Dunkl 2013), or by process mining (Bose et al. 2013). Subjectoriented BPM (S-BPM) seeks to assist this process by providing a methodology that presents process models in a manner analogous to natural language features, namely, subject, object and predicate constructs from the stakeholder's perspective (Fleischmann et al. 2012). This enables users to be engaged more effectively via a simple and intuitive process representation and via the implementation of usercentred elicitation hardware and software.

The goal of the proposed research was to use 3D virtual worlds as a means of extracting process information from stakeholders in line with S-BPM concepts. Rather than use traditional modelling elicitation techniques which heavily tax the analyst (such as interviews), or using an abstract representation and interface, such as the Metasonic touch (Oppl and Stary 2011), QUT wished to explore the idea of using 3D virtual world models of workplaces as elicitation environments. Such an approach was designed with the intention to reduce the training time and complexity of modelling by providing a more natural modelling interface. To achieve this goal, a 3D virtual world was constructed which closely matched a typical office environment. Users were then able to interact with objects in this virtual world to complete tasks as they normally would in reality. As users perform actions within the virtual world, a process model begins to develop automatically, thus the term model as you do. Once the process is completed, the model can be exported and given to analysts without stakeholders ever needing to understand the underlying grammar of the model. An overview diagram of this concept is shown in Fig. 7.1. 

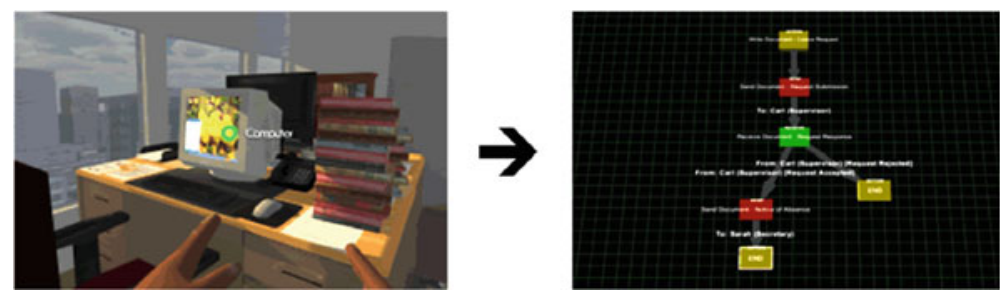

Fig. 7.1 Image of the overall approach. The virtual world on the left is used to specify processes that emerge as S-BPM grammars on the right

If this virtual world can be easily learnt, it should allow for much faster creation of processes. In addition to this, situated cognition theory suggests that the generated models should potentially have greater accuracy than what could normally be achieved with common interview techniques. Reducing the strain on external analysts would also enable many more people to be consulted about processes. Rather than have process interviews become a bottleneck for elicitation, this tool could be deployed online to allow for hundreds of people to all build process components simultaneously.

Having an easy to learn, and deployable, elicitation tool would also enable businesses to have a platform for engaging with customers during process specification. Rather than business estimating customer viewpoints, customers could directly specify exactly how they want to perform their tasks within a process. This may result in developed processes more closely matching customer expectations.

The rest of this chapter details the research, implementation and usability testing that was performed with Metasonic to meet the previously specified research goals. Section 7.1 covers the underlying reasons and processes that led to the development of the tool, including meetings with Metasonic. Section 7.2 details the actual tool developed. Section 7.3 details a trip from Brisbane to Pfaffenhofen to visit Metasonic, where QUT staff tested the tool with Metasonic staff. The chapter concludes with a discussion around Metasonic staff responses, and provides recommendations for other companies seeking to use virtual worlds for similar projects in process modelling.

\subsection{Theoretical Inspirations from Readings Dec. 2013 to Jan. 2014}

As part of the preparation process for developing the new approach, a literature review of the field was performed by the student, Joel Harman, to provide a theoretical context for the development processes, in particular, enhancing customer involvement with the development of business processes. 


\subsubsection{Customer Involvement in BPM}

While there is debate over the exact definition of BPM and its associated goals, there is a strong consensus that BPM should enable companies to adjust business processes rapidly to meet the ever changing demands of customers (Vom Brocke and Rosemann 2010). With this in mind, it is critical that the customers be accurately considered when building process models (Margaria et al. 2012). Towards this end, S-BPM is a process modelling language that was designed specifically for use with process stakeholders. The goal of this language is to simplify the grammar to a level that could easily be taught (Fleischmann et al. 2012). With this simplicity, it is surmised that businesses could involve a larger portion of their staff in the modelling process and rely much less on external analysts for support. S-BPM was designed specifically for process stakeholders, and rather than focus on completeness, it instead aims for simplicity. Processes are modelled by connecting only three types of commands: internal actions, sends and receives (Fleischmann et al. 2012). S-BPM is based on the principle of view-based modelling. Rather than build a complete model, several smaller models are constructed from the individual views of those involved in the process. The goal of this approach is to align the construction of the process much more closely with how those involved perceive it to operate (Kabicher and Rinderle-Ma 2011).

\subsubsection{Tacit Knowledge for Process Modelling}

Tacit Knowledge is the concept that not all knowledge can be easily codified. Polanyi first introduced the term with the assertion that people can know more than they can tell (Polanyi 1967). The field of knowledge elicitation ties in closely and deals with trying to work with tacit knowledge either transferring this knowledge between individuals, or converting it into encodable, explicit knowledge. There are four common methods for performing this task: interviewing experts, learning by being told, learning by seeing (Parsaye and Chignell 1988) and learning by doing (Herrgard 2000).

Interview methods are usually the most common in process modelling when working with experts. This methodology allows for the trained analyst to gather information about the process from all of the people involved in its execution and verify the process quality with respect to all parties involved. Due to the distributed nature of this approach, it has attracted significant use when working with viewbased process models (Kabicher and Rinderle-Ma 2011).

The issue with interviews is that they rely heavily on the stakeholder to provide accurate information to the analyst. In an interview setting, however, this is not always possible especially if the interviewer is unfamiliar with the field (Parsaye and Chignell 1988). Many experts forget tasks they assume to be widely known, or have difficulties explaining what they do without actually doing it (Grosskopf et al. 2010). This block on memory is commonly associated with situated cognition, the concept that knowledge is inseparable from doing (Nunberg 1978). 


\subsubsection{Situated Cognition via Virtual Worlds}

Traditional accounts of memory often focus heavily on the concept of passive storage of information. Much formal learning involves wrote-learning of information or testing of knowledge without context (Glenberg 1997). The theory of situated cognition is an alternate view on cognition which suggests that all knowledge is, to some extent, tied to the situations in which it was gained (Brown et al. 1989). This was originally considered within the context of teaching. Until this point, many believed that schools or other educational facilities were neutral environments that allowed for knowledge to be easily applied to other areas. Miller and Gildea (1987) later verified that this assumption was not true with respect to learning vocabulary. The suggested reason for this is that knowledge can only be applied to the context in which it was learned (Nunberg 1978). In language, a word is not a contained concept; words can change meaning when placed in different sentences or verbalised with different tones (Barwise and Perry 1981). The problem with this contextual information is that it cannot be encoded with standard data. While an expert may be able to explain what they do, they are unable to easily provide context to the information (Brown et al. 1989). It is suggested that the only way to accurately teach this information is to first provide this context, a practice commonly used during apprenticeships (Lave 1990). From this, theories of explicit memory (sometimes referred to as tacit knowledge) have emerged as knowledge which cannot easily be conveyed to other people. To retrieve this information, it is easiest to use a simulation-based approach for memory recall (Rubin 2006).

Jestice and Kahai (2010) back up this claim by suggesting that the reason virtual worlds prove so effective in remote learning is that they provide a level of situated cognition which cannot be achieved when operating under standard remote learning techniques. This allows for both a much more structured learning experience and the ability to learn by visiting real-life locations within the world. Leidl and Roessling (2007) have also shown that in addition to this, these worlds also improve user embodiment and experience when compared with regular external learning methods. Such research shows that we can expect greater engagement from stakeholders when using 3D virtual worlds, as we are using a visual representation that is aligned with their direct experience of work (Guo et al 2013; Brown et al. 2014).

\subsubsection{Brisbane Design Workshop Jan. 2014}

After exploring the relevant literature surrounding this project, a design workshop was carried out with staff from Metasonic (Thomas Rothschädl and Udo Kannengiesser), who visited Brisbane in Feb. 2014. The goal was to establish a potential virtual world modeller design and to identify future goals for the research work. The workshops included presentations by Thomas and Udo on their technology implementations and the theoretical bases underlying S-BPM. During the 
workshops, design concepts and prototype implementations were presented by Joel Harman and Ross Brown, and critiqued by Thomas and Udo. Those ideas were:

1. Use the Metasonic Touch to control a virtual world representation of the process model.

2. Use a stationary top-down world as an alternate interface to provide an overview of the business while using integrated S-BPM objects.

3. Execute a virtual world scenario based on an S-BPM model previously developed using the Metasonic Suite.

4. Use a first-person camera view to act out parts of a process and automatically construct a model to be exported to the Metasonic Suite.

Thomas and Udo thought that having an avatar represented in the scene was important, as it put the focus on specifying individual subject-oriented behavior, rather than a single overall model, so we decided to eliminate number two. Both QUT and Metasonic staff agreed that building the virtual world on top of the Touch device would limit its usability as a desktop PC virtual world could be handed to anyone, but touch tables would be rare, so number one was discarded. After further discussion, it was decided that the final option, number four, of using a first person camera view to act out the model would be the most rewarding choice of the four presented.

\subsection{Designing an Integrated 3D Virtual World S-BPM Approach Feb. 2014}

From the previous focus group and literature readings, we developed an approach to integrating S-BPM process elicitation into a 3D virtual world. The evidence from literature suggested that this virtual world approach would be an optimal candidate, and so a design concept was developed to be presented to the Metasonic partners at a later date in 2014.

The implementation is designed to exploit virtual world functionalities for modelling real work environments, and to enable the specification of S-BPM activities within such a world. In short, the intention is to structure the interactions with the virtual world in a similar manner to S-BPM, which has a natural language structure as the basis of its approach. We have used this interaction approach in other work (Brown et al. 2014), due to its application to a subject-oriented viewpoint in a virtual world.

3 D virtual worlds often incorporate an immersive first-person view (Weller 2007) in order to enable deep engagement with the content in question; viz. the actions taken by the viewer are from his or her own perspective. This insight has driven the research into using virtual worlds with S-BPM, as the visual metaphor facilitates a direct engagement with the fundamental concepts inherent in S-BPM, that of specifying processes from a subjective point of view (Fleischmann et al. 2012). 


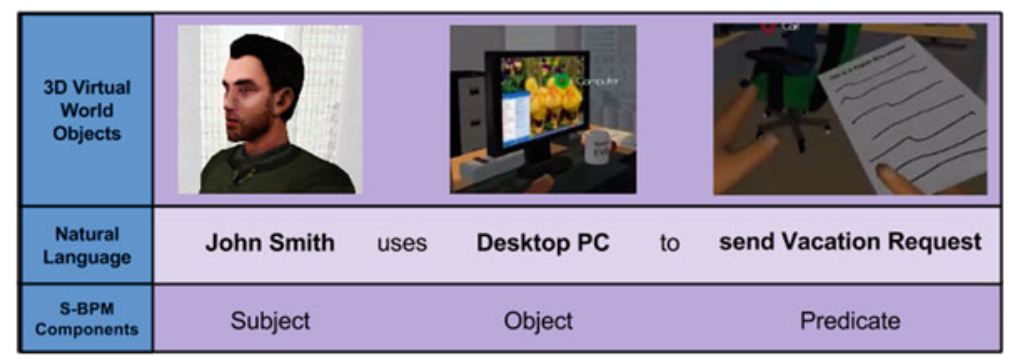

Fig. 7.2 Diagram mapping natural language constructs utilised in S-BPM onto 3D virtual world objects

This means that the concept of subject, object and predicate specifications that are the foundation of S-BPM map directly to 3D subjective virtual world interfaces. We illustrate this with a diagram showing the general S-BPM language constructs (Fleischmann et al. 2012), applied to virtual world 3D content; see Fig. 7.2.

Using a virtual world, the stakeholders can have their memory activated regarding work details and then execute their work in the environment, modelling as they do. We now show how these concepts were encapsulated and operationalised within the virtual world tool to provide an engaging interface to process modelling stakeholders.

Off-the-shelf $3 \mathrm{D}$ virtual world technology is used to provide the interactive environment to support process elicitation tasks. Such technology is ubiquitous, due to the rise of advanced graphics technology enabling virtual world and games systems to run on standard desktops. Such game engines are developed to hide (encapsulate) away underlying aspects of a game, enabling reuse of code. For example, scripts used in the environment can be uploaded into other scenes using the same engine. They also facilitate porting code to other hardware platforms, as hardware-specific factors are hidden in the central engine modules. Our implementation is created using a common proprietary game engine known as Unity3D. ${ }^{1}$ It is a completely integrated environment for the development of games which has become very popular due to its favourable licensing arrangements and its superior development technology. The major components used in the development of this application include its level design system and scripting language, Mono $\mathrm{C \#}^{2}$

The game level design system is shown in Fig. 7.3. This environment enables the uploading of $3 \mathrm{D}$ content and placement of items into the scene to be created. Once content is entered, the world can be configured by attaching scripts to the world objects, which enacts any interactions and world simulation activities. Our example application uses C\#, but other languages can be used, such as JavaScript. For our example, these scripts enable the user to move around the environment, touch objects and type text describing the tasks they have done.

\footnotetext{
${ }^{1}$ Unity3D Game Engine: www.unity3d.com, accessed August 2014.

${ }^{2}$ Unity3D Game Engine: www.mono-project.com, accessed August 2014.
} 


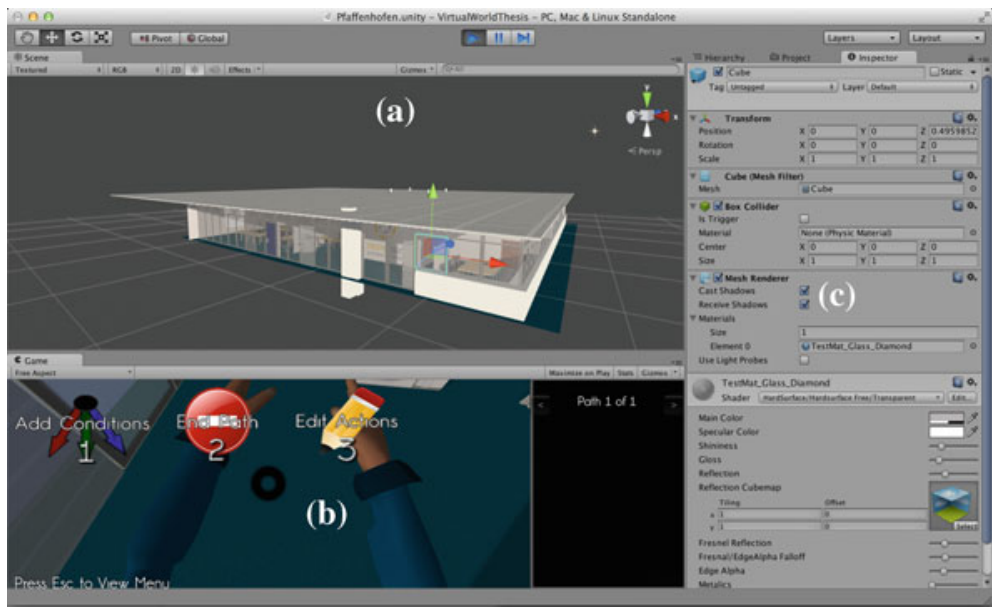

Fig. 7.3 Image of Unity3D development environment, with a level editor, $\mathbf{b}$ execution window and $\mathbf{c}$ asset management area

Using a floor plan and imported artefacts, the general layout of key areas can be quickly modelled for use in an interview scenario. For our Metasonic study, we analysed typical workspaces to determine the key objects in the rooms (e.g., chairs, tables and phones) in order to select corresponding virtual world artefacts. We argue, logically, that the objects of most relevant use should be presented at the highest level of detail as they have the most influence on the cognition of the user with respect to their process activities. We also argue that the rest of the scene may be left in lower levels of detail, with a lowered effect on the tasks being elicited. We used office artefacts gained from the Unity 3D Asset Store, ${ }^{3}$ thus minimal modelling was required for the office example tested at Metasonic. Bespoke content can also be modelled when required using typical tools such as 3DS Max. ${ }^{4}$

Once the virtual world is built, the interactions for the environment need to be scripted. Such programming is a typical part of developing 3D worlds, in a manner analogous to that of 2D widgets on standard windowed interfaces. These interaction functions support the modelling tasks undertaken within an S-BPM application. Thus, the interactions fall into two categories, human subjects and non-human objects. As per the other subject-oriented elicitation systems that have been built on the S-BPM platform (Oppl and Stary 2011), the main components that can be specified are sequences of actions, messages to other subjects and choices.

As this project is, at its essence, a process elicitation project, the $3 \mathrm{D}$ view is from a worker perspective, being an avatar-based first-person view (Burdea and Coiffet 2003). This world view provides a setting that is cognitively subjective, facilitating a personal viewpoint when eliciting to-do lists from interviewees. Objects in the virtual

\footnotetext{
${ }^{3}$ Unity3D Asset Store: https://www.assetstore.unity3d.com/en/, accessed: August 2014.

43DS Max: http://www.autodesk.com.au/products/3ds-max/overview, accessed: August 2014.
} 
world are scripted to reveal their functionality via a menu upon being clicked. The direct manipulation interface provides a natural mnemonic approach for interacting directly with the objects having most affordance for the activity (Galitz 2007; Stone et al. 2005). A further benefit of the usage of direct manipulation interfaces - also called WYSIWYG interface (what you see is what you get) - is that the objects are visible and hence the interviewees do not need to remember complex syntax (Stone et al. 2005; Hutchins et al. 1985). In this way, novices can also learn object functionalities quickly. For virtual worlds, direct manipulation principles are very helpful in providing the feeling of direct involvement with the simulation (Stone et al. 2005; Shneiderman and Plaisant 1998; Hutchins et al. 1985). Such involvement results in a more consistently defined set of activities, due to the priming interaction with a visually familiar representation of subjects and objects.

Object and subject interactions are enacted by the provision of a set of options for the object or subject being used in the action or message respectively. The actual document or artefact being exchanged between subjects is free-form text to provide the user with the flexibility to specify objects and messages. Specific steps in carrying out these interactions will now be detailed.

\subsubsection{Activity Specification}

For activity specification, the method involves the clicking on an object of interest to the execution of the action; the interface provides a mnemonic for the S-BPM approach; see Fig. 7.4.

For each object, the information recorded is drawn from embedded virtual world data, e.g., its instance name, such as "Desktop PC." A single script is used for each object, which packages the subject, object and document specification from the user,

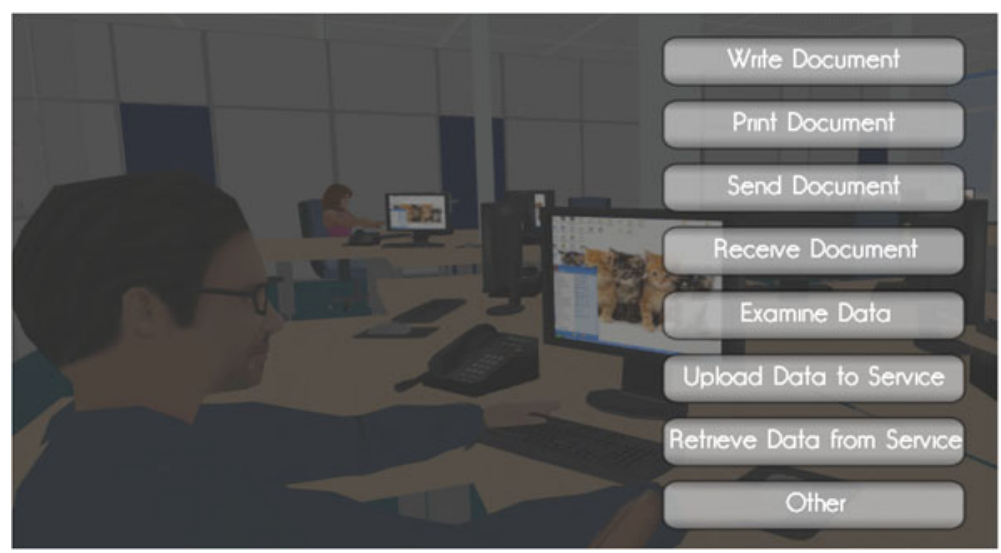

Fig. 7.4 Clicking on an object lists the actions that can be done with the object in question. In this case a desktop PC can write, print, send and receive a document, or examine, upload and retrieve data from a service 
saving it to disk. An object to be used, for example the PC shown in Fig. 7.4, has this single script attached to it via a menu interface. From then on, it is able to provide process elicitation information as text descriptions typed in by the user. This can be done for every object in the world that is relevant to the process being elicited.

S-BPM also has a message-passing construct for specifying if a person sends a message to a subject in the environment. We provide a subject overview of the environment to enable the user to identify people present in other areas of the environment. This overview prevents the person getting lost inside the environment, even if it is familiar to the user; see Fig. 7.5.

In addition, the user may specify messages sent to subjects directly in the world by traversing the environment in the first person; see Fig. 7.6.

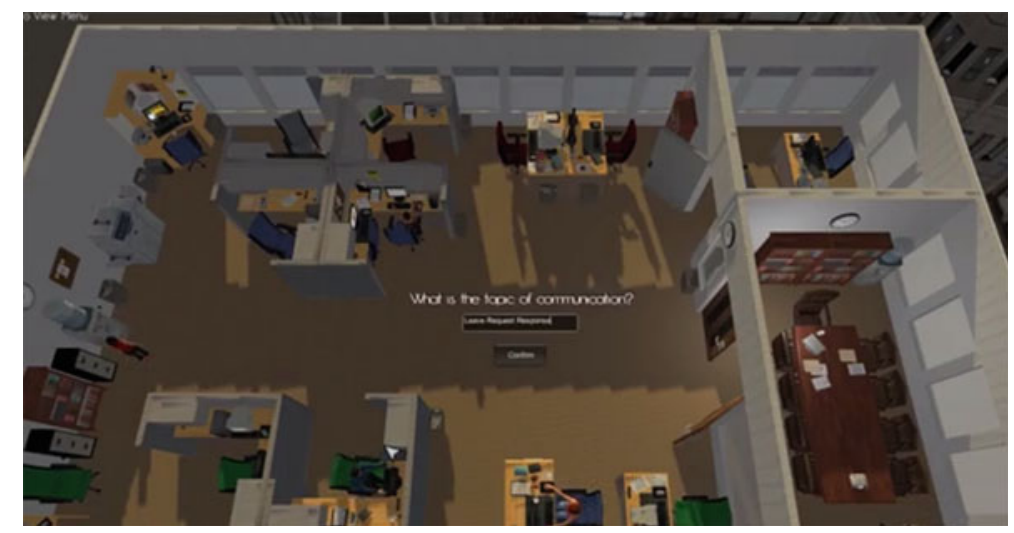

Fig. 7.5 Example using the tool to specify messages to subjects via an overview of the workplace

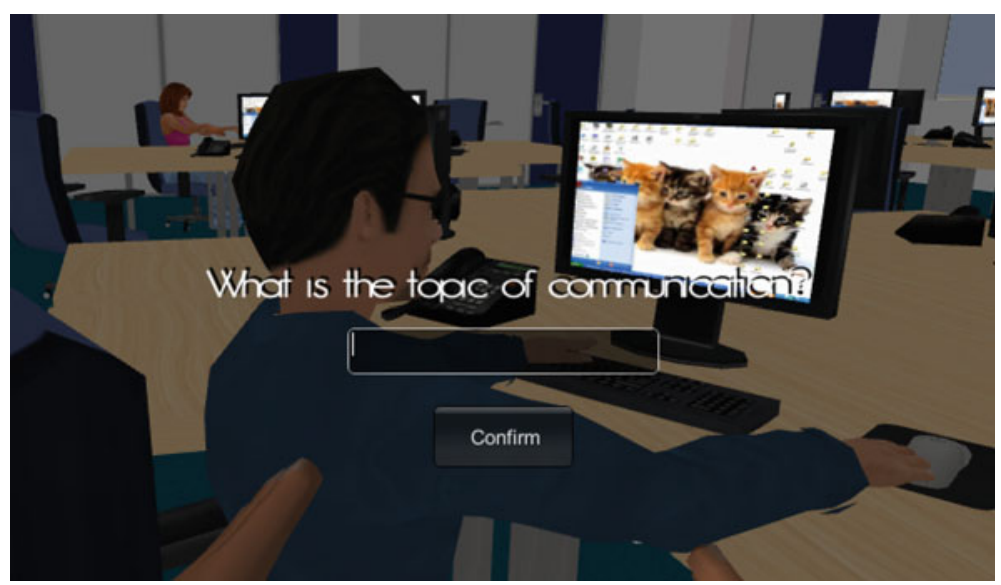

Fig. 7.6 Example using the tool to specify messages by navigating the world directly and clicking on the human subject 


\subsubsection{Choice Specification}

In addition to the specification of activities with objects and messages to subjects, we have developed a method for the specification of choice using the virtual world. This involves the use of a stepwise editor in the virtual world to go back and form a break point at the initiation of choice in the past, and then to execute the task along the new fork, as shown in the following Figs. 7.7, 7.8 and 7.9.

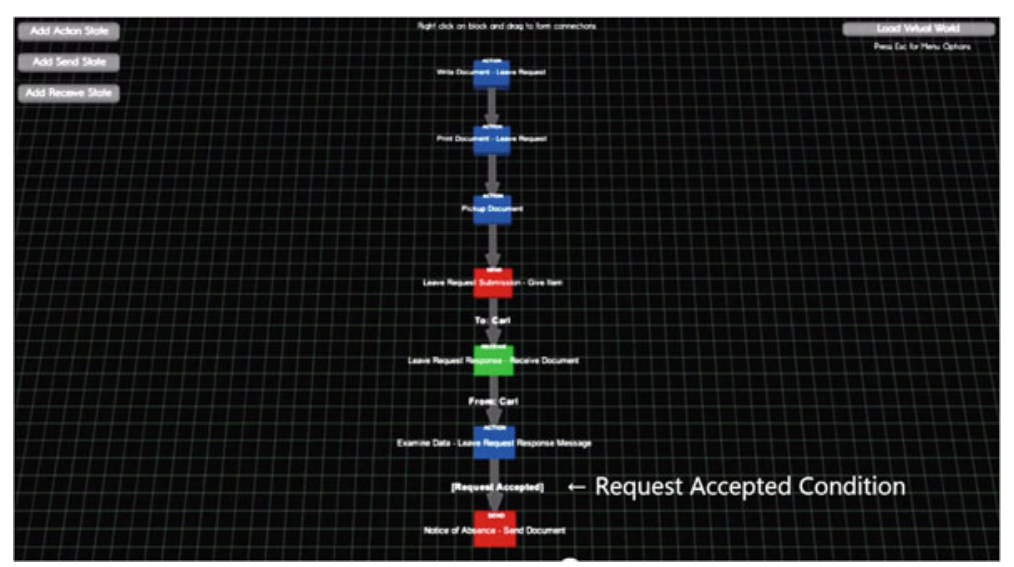

Fig. 7.7 Using the tool to specify choice in the world, showing the original S-BPM diagram, annotated to show choice insertion point

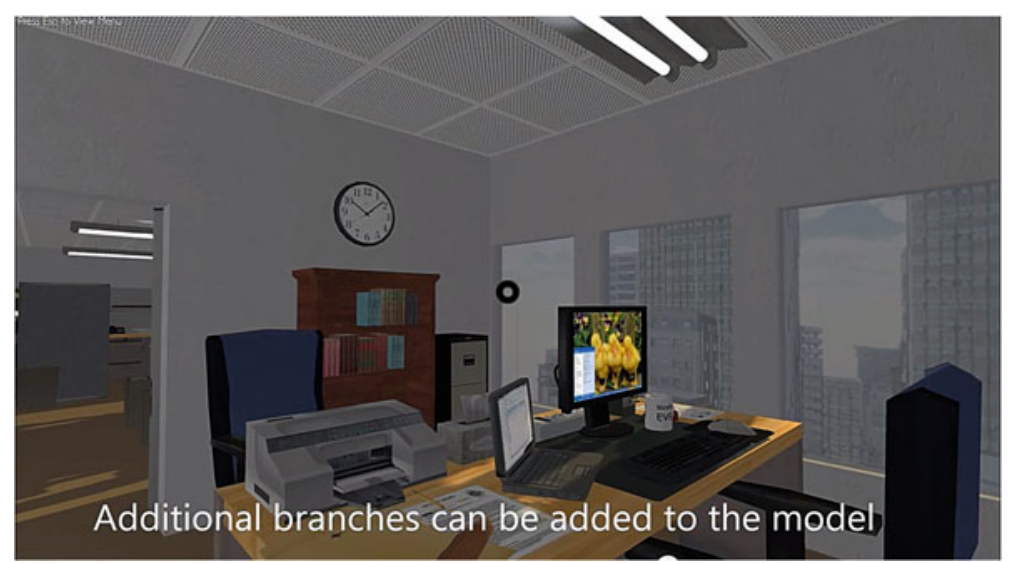

Fig. 7.8 Traversing the virtual world actions to a branch point, where the new choice is to be defined 


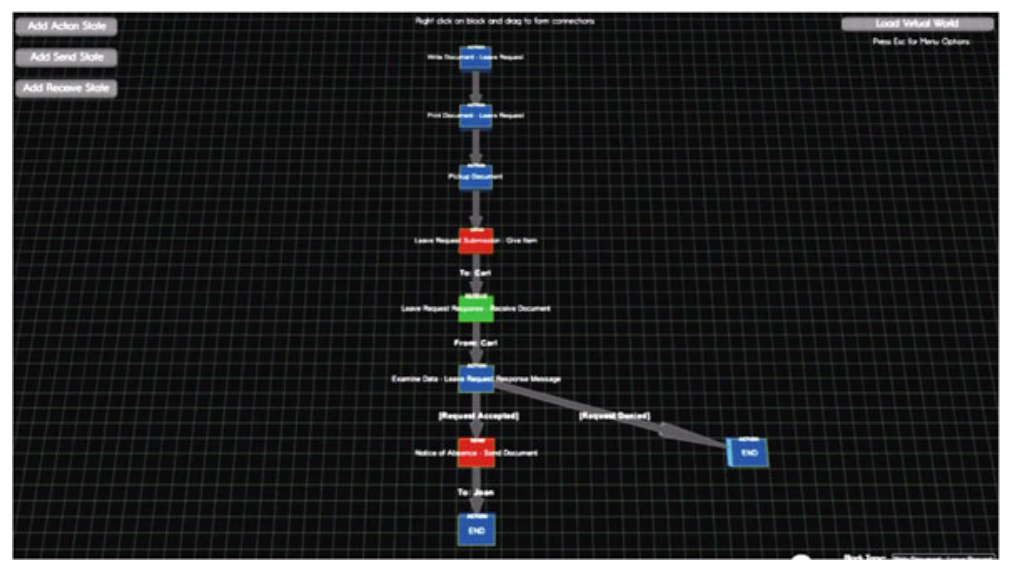

Fig. 7.9 Snapshot showing the final S-BPM diagram with a newly integrated choice after the previous interactions are performed

\subsection{Evaluation at Metasonic Headquarters April 2014}

It was agreed in April to organise a flight from Brisbane to Pfaffenhofen to strengthen the working relationship between QUT and Metasonic. The trip involved attendance at the S-BPM One conference in Eichstaett, followed by three days in Pfaffenhofen working with Metasonic. The goals for the visit were twofold. Firstly, the intention was to ascertain user acceptance of such a modelling tool with S-BPM practitioners, such as Metasonic. Secondly, is was to educate the Metasonic staff and leadership about the theoretical background to our research and provide a practical workshop in the use of the Unity 3D games engine so that Metasonic would be given a primer on strategies for using this technology in future projects. We now detail the execution of these two goals in turn.

\subsubsection{D Virtual World Tool Evaluation Approach}

The virtual world tool was evaluated using an experiment, garnering preliminary quantitative and qualitative data from Metasonic via a four step approach:

1. a short screening questionnaire, to ascertain virtual world experience levels;

2. a training video showing how to specify activities, messages and choices within the virtual world tool;

3. a test modelling scenario based upon a travel application process within a generic business;

4. a follow-up questionnaire and semi-structured interview on the usability of the virtual world tool. 
The response from Metasonic staff was encouraging, with nine people taken through the preliminary test, producing a rich set of qualitative results from participant replies. This cohort included the CEO, Herbert Kindermann, and Dr. Albert Fleischmann, a director of the company, along with software developers, sales representatives and administration staff. Overall, the response was positive to the tool, especially regarding the concept of using the virtual world to elicit process model information from the stakeholder. However, there were specific issues with the tool that were highlighted by their staff, as we now show.

\subsubsection{Quantitative Results}

Each post-test questionnaire contained 18 questions covering a number of usability factors about the virtual world tool. The questions were scored on likert scales of between one and seven, with 3.5 being the middle score. The screening test showed participants had low exposure to $3 \mathrm{D}$ virtual worlds at 1.4 , but had high modelling exposure at 5.10. Therefore, experiment participant responses are from virtual world novices, who are experienced in business process modelling. The average value for every question from the users was 5.30. This indicated a positive response, as it is above the middle value of 3.5. However, drilling down on first the negative and then the positive responses in detail provides a more nuanced story of the reception of the new tool:

- participants had major issues with recovery from mistakes, scoring 3.20 , and the completeness of the functionality of the system, 3.90.

- highest scores revolved around the interface of the system, with participants enjoying using the system, 5.60, and finding the interface pleasant, 5.40.

In summary, while the quantitative results are only preliminary in a scientific sense, the tool and approach have been marked as promising by participants. Some key issues were found in dealing with errors and a perceived lack of completeness to the functions in the system. We now list some of the more important observations by Ross and Joel as experimenters, and the comments of participants during the post-test interview.

\subsubsection{Experiment Observations}

- After thinking about a task, sometimes participants would forget which tasks they had already entered. This was commonly caused by participants being unable to remember all of the scenario at once.

- Many participants tried to complete actions from other subjects. After one person in the office sent a document, they wanted to specify actions for how the receiver should analyse it. 
- A lot of the participants spent a great deal of time looking around and exploring the scene.

- Participants would sometimes get lost in a scene and would be unable to find what they were looking for, potentially symptomatic of using a generic office for the testing.

- Participants couldn't recover easily from mistakes. When they would perform an incorrect action, many would usually continue through the scenario regardless.

- A number of participants took the "lazy" path and did all of their messaging and work from a computer. The implication here is that many business processes may be enacted completely from a computer, so such an approach is valid.

\subsubsection{Participant Comments}

- Almost all the participants commented that the tool was enjoyable to use. Some even went on to say that it did not feel like they were modelling at all.

- Many of those who used the tool noted that process stakeholders and others who were unfamiliar with modelling grammar would probably enjoy the tool, as the concept was easy to grasp.

- A lot of participants commented they had issues splitting paths correctly. This was largely a tool user interface problem.

- A lot of participants wanted the scene to be a lot more interactive than it was. This was especially noted with regards to sending of messages. Users wanted responses sent back automatically by the scene.

- Some participants were uncertain which commands they should be performing (e.g., 'Give Document' vs. 'Give Information'). They were unsure if these options would have different effects.

- People were unsure if different computers would provide the same results.

- Some participants wanted an 'Inventory' or something equivalent to know what they had/could use.

- One of the few interactive items in the scene was the piece of paper. Many participants printed out the piece of paper and manually handed it to the supervisor commenting that it gave them a sense that they were performing the process.

- Many participants commented that the UI had too many buttons and they couldn't always easily do what they wanted.

- A lot of participants wanted to be able to see the model created in realtime. In particular, a lot of them wanted to be able to see the model they were constructing. This could be due to their heavy modelling experience.

- Many wanted additional feedback when they had successfully completed a task. In particular, they wanted the world to change in some way.

- Some participants liked being able to have the choice to do the same thing in different ways (e.g., message on a computer, make a phone call, talk in person). 
- Many thought that it was very interesting to use, but some thought that it may be a novelty that quickly becomes monotonous when used repeatedly.

- Participants commented this would be especially helpful for spatially separated tasks, but less useful in largely online tasks.

\subsubsection{Games Engine Workshop}

After the previous experimental assessment of the system by Metasonic staff, the QUT researchers presented a workshop day on both the research background, presented in Sect. 7.2, and the Unity 3D technology used to generate the elicitation tool. A large number of Metasonic staff, approximately 30, attended the workshop.

The morning presentation consisted of descriptions of the theory behind this work, and an elucidation of two projects being run by Ross Brown in this research domain, including one with the University of Vienna (Brown et al. 2014).

In the afternoon, both Ross and Joel presented an introduction to games engine technology, and their instantiation in Unity3D. Ross presented a small introductory primer on game engine theory and technology, introducing some of the major features of Unity3D, especially with reference to how it is used to create interactive 3D worlds. Joel then presented a workshop session in the afternoon on how to use the level design tools and the scripting interface to generate the functionality inherent in the demo described in Sect. 7.3.

To further emphasise the ease of developing such virtual worlds using modern tools, Joel developed a model of the Metasonic headquarters (see Fig. 7.10). A convincing model was created quickly, the morning after visiting the business, in roughly three hours. This answered the often asked question regarding virtual worlds, viz. the effort required to model such environments. This was surprising and informative to the staff of Metasonic, as they realised that such tools were within the bounds of budget resources for process modelling projects due to their ability to speedily model a business.

\subsubsection{Metasonic Staff Reflections}

Metasonic staff members now note their reflections on the process of designing the tool and learning about the potential of such game engine technologies.

Nils Meyer, Metasonic CTO Business objectives are typically achieved by a close collaboration of different individuals. Taking this as a basis, the ideas of a subject- or communication-oriented view on business process models have been developed. Taking this on from a model perspective to a tool perspective is a consequent next step that fits nicely with the 3D virtual world approach.

Many practitioners today know 3D virtual worlds from gaming and very often as well from multi-player gaming. Objectives in a multi-player game can often be achieved by the close collaboration of different individuals being similar to the achievement of real-world objectives. From that perspective a $3 \mathrm{D}$ virtual world 

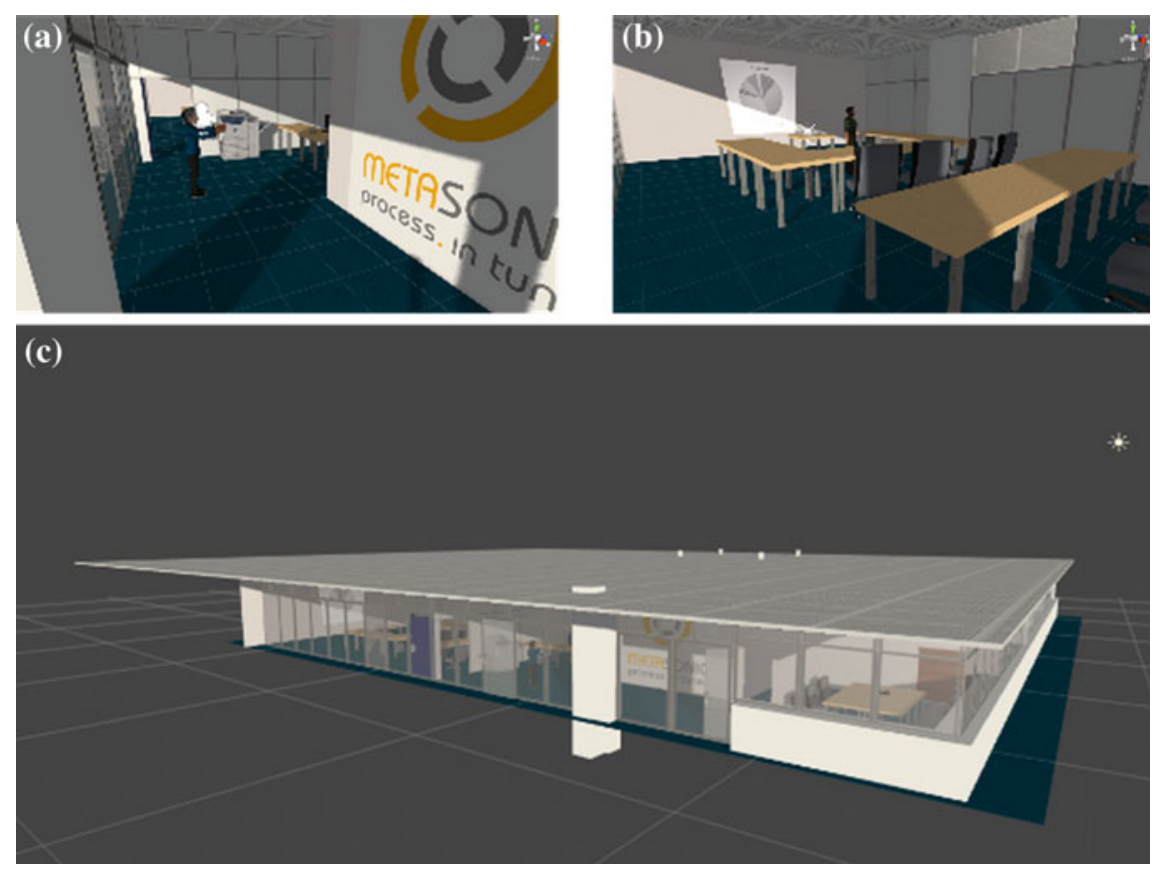

Fig. 7.10 Images taken from the Metasonic modelling exercise, built in a morning's work from photos and a floor plan. Image $\mathbf{a}$ is the reception area, $\mathbf{b}$ the meeting room and $\mathbf{c}$ is an overview of the entire building. In real life, the Metasonic headquarters is $536 \mathrm{~m}^{2}$ in floor area, housing 35 employees

approach for subject-oriented process model elicitation seemed to be and turned out to be a nice match.

The work carried out so far illustrated already some potential, especially for people not being trained in using an abstraction of real life represented by business process models. Further developments can make this way of modelling even more collaborative, easing some things being experienced now as difficult in the tool. If different individuals really could work in the same virtual world 'online' at the same time, meeting each other and observing each other, no questions on 'how should I proceed after I send the information to my colleague $X^{\prime}$ 'would occur, as I could see that he's still working on my answer. I could just wait and as soon as he's coming back to me, continue working.

Udo Kannengiesser, Metasonic R\&D Department One of the strengths of the $3 \mathrm{D}$ elicitation tool is that it closes the gap between abstract business processes and more concrete work contexts. The richness of the 3D representation cues more detailed memories of how the work is performed, thus leading to more accurate process models. Another advantage of having such a broad range of contextual information available in the $3 \mathrm{D}$ world is that it helps identify opportunities for process improvement. This is more difficult to achieve when all you have is an abstract process model. It would be interesting to apply this elicitation tool to 
domains where interactions are more physical than in typical office settings, where you interact mostly with computers and printers. This would include shop floor and logistics processes, where people operate machines, unload trucks, move boxes, drive vehicles, etc. I expect there would be an even greater impact of the tool in these domains.

The tool is built in a way that nicely integrates with key ideas of S-BPM. In subject-oriented modelling, process participants need to model only their own subject behaviour, while the behaviour of other subjects remains opaque. This is well supported by the first-person, "subjective" view of the avatars, which lets you model only the behaviour of your own avatar (i.e., your subject), and no one else's. The third-person view that is implemented in the tool lets you visualise interactions between subjects, but does not allow modelling subject behaviours from an "omniscient" perspective, as would be the case in other modelling approaches such as BPMN.

Another distinguishing concept of S-BPM that is indirectly supported by the tool is the notion of process validation. In S-BPM, processes are validated by subjects that "play" through their work steps and their interactions in a "try-out" environment before executing them in the real world. The virtual environment provides a stage for this role play, and the avatars provide the characters. The only difference is that validation is no longer performed after, but during, process modelling. So the tool realises not just "model as you do" but also "validate as you model."

Thomas Rothschädl, Metasonic R\&D Department During the development of a first version of the tool, regular video conferences helped to create a common picture of the tool. As the first results looked very promising, it was necessary to involve a broader branch of different stakeholders such as S-BPM consultants, software developers and sales representatives to obtain feedback on how the first version is perceived and where future improvements should be focused. This also enabled a deeper knowledge exchange between Metasonic employees and QUT researchers according to the S-BPM methodology and 3D virtual world creation.

The evaluation and first tryouts of the tool brought very valuable results. Participants all saw great potential, and also enjoyed, the revolutionary new way of creating a business process model through performing them in a virtual world. Most of them saw this work at this early stage already as extraordinarily valuable. Nevertheless, some modelling experts missed a visual overview of the created process (in the S-BPM modelling language). Surprisingly, people who are not experienced business process modellers also claimed that an overview of already performed tasks and message exchange would be valuable for them.

Most of the practitioners (including me) had the biggest problems with defining different branches (choices) in the way to act within a process. For example, a person asks the manager if it is okay to take a vacation, and already knows that the manager can say yes or no. Users also want to define this choice after asking the manager, although only one path can be defined in one instance. Additionally, in my view, it would be beneficial to extend the tool to enable multiple users to interact within the virtual world. 
The second day of the games engine workshop showed that, with some practice and experience in programming, the mapping of a real world into a virtual world model, based on a floor map and some photos, can be done in a very fast manner with current tools like Unity3D. From my view, this is one important requirement for showing that $3 \mathrm{D}$ virtual world modelling can be quickly used to define processes which happen in the real world.

Altogether, the productive "real-world" presentations, meetings and discussions in Pfaffenhofen enabled future improvement to the prototype and helped Metasonic and QUT to continue to pursue their close work on this topic.

\subsubsection{Notable Program Changes}

Upon returning to Australia, the QUT research team analysed the above responses from Metasonic and have implemented the following changes.

- A start-up screen has been added informing users they are only working from their point of view, to avoid any confusion on modelling other subjects' work.

- A list of actions has now been added in a side viewport, to prevent users from forgetting the previous work they have defined.

- Some participants were unsure what to do when they finished a branch. An "end" action has been added to give the user the ability to end the branch intuitively.

- A lot of participants had problems with conditions and branching. Many participants wanted to add all their conditions at once. This has now been allowed.

- Many users were waiting after they sent a message. They were expecting the recipient to send them a message back, as in a process simulation. This problem has temporarily been solved by asking the user if they want a message immediately sent back via an explicit dialogue.

\subsection{Conclusions}

Overall, the process of engagement between QUT and Metasonic over this new technology can be considered a success. Despite the risk of such an engagement not working, due to the innovative nature of the technology being used, there were significant benefits in both technological innovation, business relationships and development of insights into new research fields for Metasonic.

Research-wise, the design, implementation and evaluation of the new 3D tool provided useful research outcomes for both QUT and Metasonic. The tool proved usable and engaging, with a positive sentiment from the Metasonic staff during evaluation. Much useful development information was derived from the usability experiments, providing us with pointers to the list of improvements we have made to the tool. 
Relationship-wise, the visits by staff from Brisbane and Pfaffenhofen had the benefit of forming a strong working relationship between a university and a company widely geographically separated. Joel and Ross were able to engage with a company performing S-BPM projects for companies as significant as Hitachi, Japan. Metasonic was able to obtain insight into novel approaches to modelling, and to have an understanding of the latest games engine development technology to assist with the future use of such 3D virtual world tools in their innovation plans.

\subsection{Implications}

A number of key implications for researchers and practitioners of BPM can be derived from analysis of the previous descriptions of the project experience.

Such an engagement benefits from early interactions on design and research direction factors. While this has often been mentioned in other research, it can be stated here that the close working relationship enabled a company to more easily focus the work on relevant topics, and to enhance trust that QUT staff would deliver a good research outcome.

More specifically, with such leading edge projects it is important to give companies an early insight into the possible solutions. QUT has a depth of talent in the area of 3D game development, and so was able to provide a very early prototype. This early prototype facilitated buy-in by Metasonic. A key insight is therefore to prototype early and often. Present game engine tools like Unity3D allow this to occur, facilitating easier insights into future innovation possibilities.

Such relationships can provide many insights into new technologies and research for companies. Metasonic had little experience in the area of 3D games and virtual worlds. This project has given them an assessment of its practical usefulness for their business, and importantly, how hard it is to implement such ideas with present tools. In particular, Metasonic appreciated the construction of a model of their Pfaffenhofen office by QUT staff as an example of the capabilities of the technology. We view such a practical exemplar close to the experience of Metasonic as being a strong point of connection for their staff, helping overcome opposition to the new approaches presented. We recommend finding such touch points in research engagements, in order to ease any resistance to new ideas.

Acknowledgments The authors acknowledge the financial assistance of Metasonic $\mathrm{GmbH}$ in the development and evaluation of the virtual world tool in this project.

Open Access This chapter is distributed under the terms of the Creative Commons Attribution Noncommercial License, which permits any noncommercial use, distribution, and reproduction in any medium, provided the original author(s) and source are credited. 


\section{References}

Barwise J, Perry J (1981) Situations and attitudes. J Philos 78:668-691

Bose R, Mans R, van der Aalst W (2013) Wanna improve process mining results? In: 2013 IEEE symposium on computational intelligence and data mining (CIDM), pp 127-134

Brown JS, Collins A, Duguid P (1989) Situated cognition and the culture of learning. Educ researcher 18(1):32-42

Brown R, Rinderle-Ma S, Kriglstein S, Kabicher-Fuchs S (2014) Augmenting and assisting model elicitation tasks with 3D virtual world context metadata. In: Meersman R, Panetto H, Dillon T, Missikoff M, Liu L, Pastor O, Cuzzocrea A, Sellis T (eds) On the move to meaningful internet systems: OTM 2014 conferences, vol 8841. Lecture notes in computer science. Springer, Berlin, pp 39-56

Burdea G, Coiffet P (2003) Virtual reality. Wiley, New York

Dunkl R (2013) Data improvement to enable process mining on integrated non-log data sources. In: Moreno-Daz R, Pichler F, Quesada-Arencibia A (eds) EUROCAST 2013, LNCS, vol 8111, Springer, Heidelberg, pp 491-498

Fleischmann A, Schmidt W, Stary C, Obermeier S, Boerger E (2012) Subject-oriented business process management. Springer Publishing Company, Heidelberg

Galitz WO (2007) The essential guide to user interface design: an introduction to GUI design principles and techniques. Wiley, New York

Glenberg AM (1997) What memory is for: creating meaning in the service of action. Behav Brain Sci 20(1):41-50

Grosskopf A, Edelman J, Weske M (2010) Tangible business process modeling methodology and experiment design. In: Rinderle-Ma S, Sadiq S, Leymann F (eds) Business process management workshops, vol 43. Lecture notes in business information processing. Springer, Berlin, pp 489-500

Guo H, Brown R, Rasmussen R (2013) A theoretical basis for using virtual worlds as a personalised process visualisation approach. In: Franch X, Soffer P (eds) CAiSE workshops 2013, LNBIP, vol 148. Springer, Heidelberg, pp 229-240

Herrgard T (2000) Difficulties in diffusion of tacit knowledge in organizations. J Intellect Capital 1 (4):357-365

Hutchins EL, Hollan JD, Norman DA (1985) Direct manipulation interfaces. Hum-Comput Interact 1(4):311-338

Jestice R, Kahai S (2010) The effectiveness of virtual worlds for education: an empirical study. In: Proceedings of the sixteenth americas conference on information systems (AMCIS), association of information systems. http://aisel.aisnet.org/amcis2010/512

Kabicher S, Rinderle-Ma S (2011) Human-centered process engineering based on content analysis and process view aggregation. In: Mouratidis H, Rolland C (eds) Advanced information systems engineering, vol 6741. Lecture notes in computer science. Springer, Berlin, pp 467-481

Lave J (1990) The culture of acquisition and the practice of understanding. In: Stigler JW, Schweder RA, Herdt G (eds) Cultural psychology. Cambridge University Press, Cambridge, pp 309-327 (Cambridge Books Online)

Leidl M, Roessling G (2007) How will future learning work in the third dimension? In: Proceedings of the 12th annual SIGCSE conference on innovation and technology in computer science education. ACM, New York, ITiCSE'07, pp 329-329

Margaria T, Boßelmann S, Doedt M, Floyd B, Steffen B (2012) Customer-oriented business process management: vision and obstacles. In: Hinchey M, Coyle L (eds) Conquering complexity. Springer, London, pp 407-429

Miller GA, Gildea PM (1987) How children learn words. Sci Am 257:94-99

Nunberg G (1978) The pragmatics of reference. Indiana University Linguistics Club, Bloomington 
Oppl S, Stary C (2011) Effects of a tabletop interface on the co-construction of concept maps. In: Campos P, Graham N, Jorge J, Nunes N, Palanque P, Winckler M (eds) Human-computer interaction INTERACT 2011, vol 6948. Lecture notes in computer science. Springer, Heidelberg, pp 443-460

Parsaye K, Chignell M (1988) Expert systems for experts. Wiley, New York

Polanyi M (1967) The tacit dimension. University of Chicago Press, Chicago

Rubin DC (2006) The basic-systems model of episodic memory. Perspect Psychol Sci 1(4):277-311

Shneiderman B, Plaisant C (1998) Designing the user interface: strategies for effective humancomputer interaction, 4th edn. Addison-Wesley Longman, Boston

Stone D, Jarrett C, Woodroffe M, Minocha S (2005) User interface design and evaluation. Morgan Kaufmann, Los Altos

Vom Brocke J, Rosemann M (2010) Handbook on business process management. Springer, Heidelberg

Weller M (2007) Virtual learning environments: using, choosing and developing your VLE. Routledge, London 